\title{
CALCULATION OF OSCILLATORY EXCHANGE IN bcc-Fe/Cu MULTILAYERS
}

\author{
S. KROMPIEWSKI \\ Institute of Molecular Physics, Polish Academy of Sciences \\ Smoluchowskiego 17, 60-179 Poznań, Poland \\ J. Pirnay, U. Krey and F. Süss \\ Institute of Physics III, University of Regensburg, Germany
}

\begin{abstract}
Within a tight-binding LMTO approach in the atomic sphere approximation we calculate the exchange interaction, $J$, between magnetic Fe slabs in a periodic multilayer arrangement $m \mathrm{Fe} / n \mathrm{Cu} / \ldots$ We concentrate on cases, where $\mathrm{Cu}$ adapts to the bcc structure of Fe. For the "open" (100)-interface our results suggest that a minimal number of $m=13 \mathrm{Fe}$ monolayers per Fe slab is necessary to get antiferromagnetic coupling. For $m=16$ we find antiferromagnetic coupling for $8 \leq n \leq 14$, i.e. a long period of $c a .11$ and a short period of $2 \mathrm{Cu}$ monolayers. In contrast, for the (110)-interfaces, only a long period of 14 monolayers is found, i.e. the coupling is antiferromagnetic for $7 \leq n \leq 14$ already for small values of $m$. We then model the roughness by mutual interchange of $50 \%$ of the $\mathrm{Fe}$ and $\mathrm{Cu}$ atoms, respectively, in the interface layers and find that, due to the interchange, the coupling constant $J$, as a function of the Cu-spacer thickness $n$, roughly shifts by half a monolayer to the right. In the (100)-case, the short period gets washed out by the roughness.
\end{abstract}

PACS numbers: 75.70. Ak

\section{Introduction}

Recently there is considerable interest in the magnetism of layered metallic systems consisting of thin slabs of ferromagnetic material separated by nonmagnetic or antiferromagnetic material, prepared either in a periodic or a trilayer arrangement, e.g. of the type $m \mathrm{Fe} / n \mathrm{Cu} / m \mathrm{Fe}$, where $m$ and $n$ denote the number of magnetic and nonmagnetic monolayers, respectively. In these systems, one observes among other phenomena an exchange-interaction between the magnetic slabs, which depends on the number $n$ in an oscillatory and slowly decaying way, with sometimes two or more periods, depending on the considered system [1].

In the present paper we study the oscillatory exchange numerically for periodic bcc- $m \mathrm{Fe} / n$ Cu-multilayers and find a drastic difference for (100) and (110) 
interface orientations. For the $(100)$ case we observe two oscillation periods, a short one of $2 \mathrm{Cu}$ monolayers and a prominent longer period of $11 \mathrm{Cu}$ monolayers, whereas for the (110) orientation only a long period of 14 monolayers is found. Surprisingly, we find additionally that for the case of (100), in contrast to the (110) case, a minimum number $m_{\mathrm{c}}$ of $13 \mathrm{Fe}$ monolayers is necessary for the appearance of an antiferromagnetic interaction.

\section{Computational method}

We use a tight-binding LMTO calculation in the atomic sphere approximation (ASA) with the local exchange-correlation potential of Barth and Hedin [2]. Since we have already described our method in Ref. [3], we only mention the following. In this method, which is particularly suited for large systems and can even be applied for systems with disorder, both the so-called "structure matrices" determining the hopping of an electron between different atomic spheres, and also the potential parameters determining the wave functions within the spheres, are determined self-consistently from the "first principles" for all orbitals up to the $3 d-, 4 s-$ and $4 p$-orbitals. Within the calculation a continued-fraction evaluation is used, with the termination procedure of Beer and Pettifor [4]. We have used 25 and 50 atoms in the basic plane of our clusters for the (100) and (110) cases, respectively, with periodic boundary conditions in all directions, using up to 60 planes in the antiferromagnetic cases, for crystallographic unit cells with $n+m$ up to 30 . The number of pairs of continued fraction coefficients was tuned to the size of the system, i.e. 5,6 , and 10 pairs were calculated before termination for $s$-, $p$ and $d$-states, respectively.

\section{Results}

The exchange-coupling energy $J(n, m)$ per interface area is defined through the equation $2 J(n, m) A=E_{\uparrow \downarrow}-E_{\uparrow \uparrow}$, where the energy difference $E_{\uparrow \downarrow}-E_{\uparrow \uparrow}$ between the antiferromagnetic and ferromagnetic states refers to the crystallographic unit cell containing $n+m$ atoms, and $A$ is the area of the basic square of this cell. For (100), in this plane, our structure had the original lattice constant of bcc $\mathrm{Fe}$, whereas for the (110) interface that of fictitious bcc Cu was used.

We first performed systematic studies of bcc-(100) $4 \mathrm{Fe} / n \mathrm{Cu}$ multilayers, with $n=3, \ldots, 10$, as well as additional studies of $m \mathrm{Fe} / 10 \mathrm{Cu}$ with $m=$ $4, \ldots, 16$, in order to evaluate the role of the amount of Fe layers. Then, for this orientation we have studied the multilayer $16 \mathrm{Fe} / n$ Cu for $n=6, \ldots, 14$. The last-mentioned structure is close to the trilayer structure studied experimentally by Celinski and Heinrich [5]. We have found, see [6], that the exchange can be split into separate contributions resulting from the $\mathrm{Fe}$ and the $\mathrm{Cu}$ spheres. Surprisingly, the $\mathrm{Cu}$ contribution apparently always favours ferromagnetic coupling of the Fe slabs. The reason may be that the electronic energy of the $\mathrm{Cu}$ segments is alowered by the indirect interaction mediated through $\mathrm{Fe}$. This lowering is more effective for parallel alignment of adjacent Fe slabs, due to a better "matching" of the electronic states. However, this tendency to favour ferromagnetic alignment 
is overcompensated by an opposing tendency from the Fe contribution: i.e. if $m$ becomes larger than $m_{c}=13$, then antiferromagnetic exchange appears.

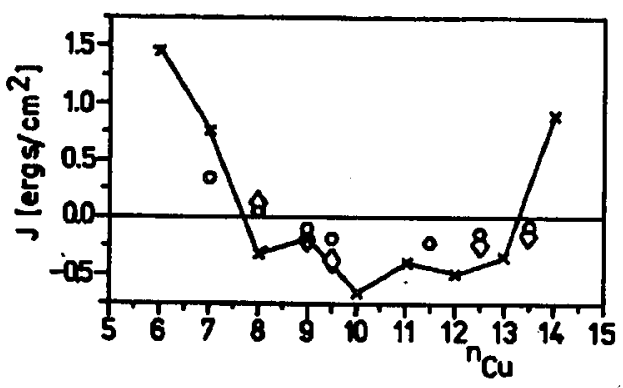

Fig. 1. Exchange energy constant $J=0.5 \Delta E /(2.87 \AA)^{2}$ for bcc-(001) $16 \mathrm{Fe} / n \mathrm{Cu}$ multilayers (numerical calculation, $(x), T=0 \mathrm{~K}$ ) and experimental results from [5] for $5.7 \mathrm{Fe} / n \mathrm{Cu} / 9.7 \mathrm{Fe}$ trilayers, for $T=295 \mathrm{~K}$ (open circles) and $T=77 \mathrm{~K}$ (open diamonds).

This is shown in Fig. 1, where for $m=16$ the dependence of $J(n)$ on the number $n$ of $\mathrm{Cu}$ layers is presented. We find ferromagnetic coupling for $n \leq 7$ and $n \geq 14$, and antiferromagnetic coupling in between. Moreover, besides the period of $11 \mathrm{Cu}$ monolayers, we also find a short period of roughly two monolayers. On the other hand, in Fig. 2, for the (110) case, only a long period of roughly 14 monolayers is present. In that case, for a bcc-(110) $8 \mathrm{Fe} / n \mathrm{Cu}$. multilayer, there is antiferromagnetic ordering for $7 \leq n \leq 14$, without short range oscillations. In this case we did not find a minimum number of $\mathrm{Fe}$ monolayers.

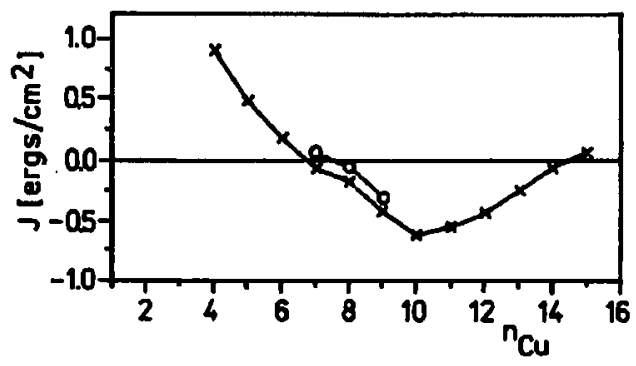

Fig. 2. Numerical results for the exchange energy constant at $T=0 \mathrm{~K}$ for bcc-(110) $8 \mathrm{Fe} / n \mathrm{Cu}$ multilayers. The crosses represent the results for smooth interfaces, whereas the circles are for interfaces, where at the interface layers $50 \%$ of $\mathrm{Fe}$ atoms are replaced by $\mathrm{Cu}$ and vice versa. 
We also performed calculations for non-ideal interfaces by modelling the roughness by mutual interchange of $50 \%$ of the $\mathrm{Fe}$ and $\mathrm{Cu}$ atoms, respectively, in the interface layers. From Fig. 2 we find that $J$, as a function of $n$, roughly shifts by 0.5 monolayers to the right, compared with the ideal interface. In the (100)-case, the roughness makes the short period vanish. Experimental points from Celinski and Heinrich [5] are also presented in Fig. 1. These authors performed measurements of $J(n)$ on a trilayer system $m_{1} \mathrm{Fe} / n \mathrm{Cu} / m_{2} \mathrm{Fe}$, for two different temperatures, $T=77 \mathrm{~K}$ and $T=295 \mathrm{~K}$, and from Fig. 1 it seems that the experimental points would extrapolate to our results for $T \rightarrow 0$.

The different behaviour of the (100) and (110) cases can partially be understood on the basis of the RKKY approximation [1], namely with the help of Fig. 3 in [7], where Johnson et al. discuss experiments on bcc-(100) $\mathrm{Fe} / n \mathrm{Cu} / \mathrm{Fe}$ trilayers by comparison with the Fermi surface of (fictitious) bcc-Cu. These results are essentially in agreement with those of [5], and from the topology of the Fermi surface in [7] one can also understand that with the (110) orientation, in contrast to the (100)-case, only one long period should be seen.

Thus it remains to be seen whether our theoretical result of a minimum nuriser of Fe monolayers, which we find for the more "open" (100) interface, but not for the (110) case, will be seen experimentally.

\section{Acknowledgments}

The authors would like to thank the Polish Academy of Sciences and the German Ministry for Research (BMFT) for support under the bilateral project X083.0. We also thank the LRZ in Munich, the LRZ in Jülich and the Computing Center of the University of Regensburg for computing time.

\section{References}

[1] P. Bruno, C. Chappert, Phys. Rev. Lett. 67, 1062 (1991).

[2] U. von Barth, L. Hedin, J. Phys. C, Condens. Matter 5, 1629 (1972).

[3] S. Krompiewski, U. Krauss, U. Krey, J. Magn. Magn. Mater. 92, L295 (1991).

[4] N. Beer, D.G. Pettifor, in: Electronic Structure of Complex Systems, Eds. P. Phariseau, W.M. Temmerman, Plenum, New York 1984, p. 769.

[5] Z. Celinski, B. Heinrich, J. Magn. Magn. Mater. 99, L25 (1991).

[6] S. Krompiewski, J. Pirnay, U. Krey, J. Magn. Magn. Mater. 121, 238 (1993).

[7] T. Johnson, S.T. Purcell, N.W.E. McGee, R. Coehoorn, J. aan de Stegge, W. Hoving, Phys. Rev. Lett. 68, 2688 (1992). 